\title{
BMJ Open Impact of group practices on patients, physicians and healthcare systems: protocol for a scoping review
}

\author{
Terry M Zwiep, ${ }^{1}$ Joshua A Greenberg, ${ }^{1}$ Fady Balaa, ${ }^{1}$ Daniel I Mclsaac, ${ }^{2,3}$ \\ Reilly P Musselman, ${ }^{1}$ Isabelle Raiche, ${ }^{1}$ Lara Williams, ${ }^{1}$ Husein Moloo ${ }^{1,3}$
}

To cite: Zwiep TM, Greenberg JA, Balaa F, et al. Impact of group practices on patients, physicians and healthcare systems: protocol for a scoping review. BMJ Open 2018;8:e022164. doi:10.1136/ bmjopen-2018-022164

- Prepublication history and additional material for this paper are available online. To view these files, please visit the journal online (http://dx.doi. org/10.1136/bmjopen-2018022164).

Received 5 February 2018

Revised 17 July 2018

Accepted 22 August 2018
Check for updates

(C) Author(s) (or their employer(s)) 2018. Re-use permitted under CC BY-NC. No commercial re-use. See rights and permissions. Published by BMJ.

${ }^{1}$ Department of Surgery, The Ottawa Hospital, Ottawa,

Ontario, Canada

${ }^{2}$ Department of Anesthesiology and Pain Medicine, The Ottawa Hospital, University of Ottawa, Ottawa, Ontario, Canada

${ }^{3}$ The Ottawa Hospital Research Institute, Ottawa, Ontario, Canada

Correspondence to

Dr Terry M Zwiep;

tzwiep@toh.ca

\section{ABSTRACT}

Introduction Group practices have potential benefits for patients, physicians and healthcare systems. Although group practices have been around for many years, research in this area is lacking and generally is centred around the economic benefits that may be realised from group practice. The aim of this scoping review is to identify the impact that group practices have on patients, physicians and healthcare systems to guide further research in this area.

Methods and analysis A scoping review will be performed based on the methodology proposed by Arksey and O'Malley and refined by Levac and colleagues. MEDLINE, EMBASE, Cochrane Central and Cochrane Economic Database will be searched from inception to present day to identify relevant studies that assess the impact of group practices on patient care, satisfaction and outcomes; physician quality of life, satisfaction and income and healthcare systems. Titles and abstracts will be screened by two members and the abstraction results charted and verified. Qualitative and quantitative analyses will be performed to identify key themes.

Ethics and dissemination Research ethics board approval is not required for this scoping review. A consultation phase will be used to discuss the results with key stakeholders followed by dissemination at local and national levels. We will also publish the results in a peerreviewed journal.

\section{BACKGROUND}

One of the first group practices was set up by the Mayo brothers in the mid-1880s. ${ }^{1}$ Over the years, this was transformed into a large organisation. Significant benefits have been realised by clinicians and patients and The Mayo Clinic is a well-established centre of excellence. Currently, there are many physicians in the USA practising as part of a group (The Physicians Foundation 2014 Survey of American's Physicians). In a recent review focusing on group practices, the overall shift towards group practices in the USA as well as the various definitions for group practices was assessed. Future research from that review will focus on group consolidation. ${ }^{2}$ The current literature supports the overall economic

\section{Strengths and limitations of this study}

- This will be the first scoping review to assess the outcomes that group practices have on patients, physicians and healthcare systems.

- This comprehensive review of the literature will be performed by assessing multiple databases with broad search parameters based on established guidelines for scoping reviews.

- This will guide further research in the area of group practices with an aim of improving patient care.

- Limitations of this study include language restrictions.

benefits of these groups and improvements in service provision in underserviced or rural areas. ${ }^{34}$ In primary healthcare, a 2013 systematic review of group practices demonstrated a positive association between group practice and clinical processes, physician opinions and innovation. ${ }^{5}$ However, no effect was seen for patient measures.

Group practices have the potential to considerably alter the way healthcare is delivered to patients and it is important to consider this impact, especially if the group is formed for reasons that are not related to patient care. The benefit to providers is important to consider as we become more aware of the prevalence of physician burnout. ${ }^{67}$ In a recent study by Shanafelt et al, $54.4 \%$ of physicians in the USA reported at least one symptom of burnout. ${ }^{8}$ This appears to be worsening and better methods are required to address burnout. Therefore, some of the motivation for setting up a group practice may be focused on improving patient outcomes, physician quality of life, physician income or the overall functioning of a larger healthcare facility or system. Whatever the motivation, the effects of a group practice need to be considered to determine the advantages or disadvantages to healthcare providers, the populations they serve and the systems they work within. 
To our knowledge, there remains a paucity of literature, and no knowledge synthesis, specific to surgical group practices. The objectives of our study are to review the literature for evidence that explores the impact that group practices have on patients with regard to access to care, clinical outcomes and overall satisfaction compared with standard individual practices. We will also explore the impact that group practices have on physicians with regard to quality of life, job satisfaction and income. Finally, the effects on healthcare systems will also be considered and synthesised. Other outcomes of interest may be identified during this review of the literature and they will be considered and synthesised. Although our focus will be on surgical group practices, we will also include all other medical groups in order to perform a complete assessment of the literature as key concepts may be translatable between medical specialties.

\section{METHODS}

To address this knowledge gap, we propose a scoping review to summarise the impact of group practice on clinician, patient and health system outcomes. Because we expect study designs, practice types and outcome measures to be heterogeneous, we will use the scoping review methodology proposed by Arksey and O'Malley and refined by Levac and colleagues to synthesise relevant knowledge. ${ }^{910}$

\section{Stage 1: identifying the research question}

According to Levac et al, in order to properly conduct a scoping review, an appropriate research question needs to be identified and linked to a clear purpose ${ }^{9}$. This question needs to consider the concept, target population and health outcomes of interest so that the review can be focused. With this in mind, the purpose of this study is to review the literature for evidence that explores the impact that group practices have on patients with regard to access to care, clinical outcomes and overall satisfaction. We will also explore the impact that group practices have on physicians with regard to quality of life, job satisfaction and income. The effects on healthcare systems will also be considered. Our research question is as follows:

What benefits do group practices have for patients, physicians and healthcare systems?

\section{Stage 2: identifying relevant studies}

We have used the research purpose and question to guide the decision-making around the search to keep the review focused. An academic health sciences librarian was consulted and was instrumental in the development of the search strategy. EMBASE, MEDLINE, Cochrane Central and Cochrane Economic Database will be searched from database inception to present day for both quantitative and qualitative literature. No limits will be placed on the search other than the inclusion of studies in the English language. The search strategy will be checked against key references to ensure that all relevant studies are captured. The search strategy will be peer reviewed using a formal process. ${ }^{11}$ The draft search strategy used for EMBASE and MEDLINE is included in the online Supplementary appendix A and will be updated as necessary to ensure inclusion of all relevant studies. The grey literature will also be searched using OpenGrey and Google. We will review the reference lists of included studies to ensure that all relevant literature has been identified and contact authors should more information be required.

\section{Stage 3: study selection}

The titles and abstracts will be screened independently by two authors for inclusion. Full-text documents will then be obtained for studies that meet the inclusion criteria or if there is uncertainty about inclusion. The full text will be assessed by one author for final inclusion and verified by another reviewer and data will be abstracted using piloted data abstraction forms. Any disagreements about inclusion will be resolved by discussion between the two authors or the input of other authors if disagreement persists. Reasons for excluding studies will be documented. Inclusion criteria was developed based on the population, concept and context framework suggested by the Joanna Briggs Institute. ${ }^{12}$

We will include all studies that:

- Include patients receiving or clinicians providing care within any type of group practice (population).

- Assess the advantages and disadvantages of group practices (concept).

- We will examine all studies from group practices in all areas of medicine which report outcomes relevant to patients, clinicians or health system stakeholders (context).

- All study designs will be included.

We will exclude all studies that:

- Are not published in English.

\section{Stage 4: charting the data}

DistillerSR (Evidence Partners, Ottawa, Canada) will be used for screening and data extraction. Citation abstracts will be uploaded to DSR along with screening questions which will be tested based on the inclusion and exclusion criteria.

A standardised form in DSR will be used to collect data. The form will be tested on 10 included studies and modified if needed. Data abstraction will be performed by one reviewer and verified by a second reviewer. Demographic information, objectives, methods, interventions, outcomes and key findings will be recorded. As the scoping review is completed, this table may need to be updated to capture all appropriate information. This will be documented and any changes to the data collection form will be explained.

Data that will be charted will include authors, year of publication, country of origin, objectives, type and size of group practice, population studied (patients, physicians, etc), sample size, methods and type of study, interventions, outcomes and key findings. 
Stage 5: collating, summarising and reporting results

Collected data will be analysed both quantitatively and qualitatively to address the research question of this scoping review. Quantitative analysis will include assessing the frequency of populations studied as well as the types of papers that have been published on group practices. Mapping of the results and a formal qualitative analysis will be performed to identify key themes as well as gaps in knowledge. The final format for the presentation of our results will depend on the data that is gathered as well as consultation with members of the team. Due to the heterogeneity of the expected included studies, a quality assessment will not be performed. Although this causes some challenges with interpreting scoping reviews, it is unknown whether it impacts on the relevance of the findings according to Levac et al, particularly in this study which is being performed to guide further research in this area. ${ }^{9}$

\section{Stage 6: consultation}

Throughout the development of this scoping review protocol, we have consulted with other key stakeholders who are part of other group practices as well as those who have experience with scoping reviews. We will continue to obtain their feedback throughout the scoping review process. We will also be including a patient advisor in the process to ensure that we have an adequate understanding of patient priorities and perspectives with respect to this project. Prior to publication of the results and dissemination of the data, we will once again consult with these stakeholders to ensure that the results have been presented in a clear and accurate way.

\section{Patient and public involvement}

Patient involvement in this project will be very important as one of our main objectives is assessing the impact of group practices on patients. Although patients were not involved in the design of this protocol, we have already identified a patient advisor who will assist us with the actual scoping review. This will ensure that the process is adequately informed by patient priorities and experiences.

\section{ETHICS AND DISSEMINATION}

This scoping review does not require research ethics board approval. This study will be completed in August 2018. We will disseminate our results by means of a presentation at a national surgical conference. We will also publish our findings in a peer-reviewed journal. This scoping review will allow us to assess the literature for knowledge gaps that we may be able to focus future research activity on. The authors' group practice provides a unique opportunity to explore some of the questions that will be raised by this scoping review.

Acknowledgements We would like to acknowledge Risa Shorr for her help in developing the search strategy for this scoping review.

Contributors HM and FB conceived the study. TZ and HM drafted the protocol and developed the research question. JG, FB, DM, RM, IR and LW reviewed and approved the final protocol. HM is the guarantor.

Funding The authors have not declared a specific grant for this research from any funding agency in the public, commercial or not-for-profit sectors.

Competing interests None declared.

Patient consent Not required.

Provenance and peer review Not commissioned; externally peer reviewed.

Open access This is an open access article distributed in accordance with the Creative Commons Attribution Non Commercial (CC BY-NC 4.0) license, which permits others to distribute, remix, adapt, build upon this work non-commercially, and license their derivative works on different terms, provided the original work is properly cited, appropriate credit is given, any changes made indicated, and the use is non-commercial. See: http://creativecommons.org/licenses/by-nc/4.0/.

\section{REFERENCES}

1. Fye WB. Presidential address: The origins and evolution of the Mayo Clinic from 1864 to 1939: a Minnesota family practice becomes an international "medical Mecca". Bull Hist Med 2010;84:323-57.

2. Tan D, Kash B. Physician group practice trends: a comprehensive review. J Hosp Med Manag 2016;2:1-8.

3. Burns LR, Goldsmith JC, Sen A. Horizontal and vertical integration of physicians: a tale of two tails. Adv Health Care Manag 2013;15:39-117.

4. Breon TA. Rural surgical practice: an lowa group model. Surg Clin North Am 2009;89:1359-66.

5. Damiani G, Silvestrini G, Federico B, et al. A systematic review on the effectiveness of group versus single-handed practice. Health Policy 2013;113:180-7.

6. Rothenberger DA. Physician burnout and well-being: a systematic review and framework for action. Dis Colon Rectum 2017;60:567-76.

7. West CP, Dyrbye LN, Erwin PJ, et al. Interventions to prevent and reduce physician burnout: a systematic review and meta-analysis. Lancet 2016;388:2272-81.

8. Shanafelt TD, Hasan O, Dyrbye LN, et al. Changes in burnout and satisfaction with work-life balance in physicians and the general US working population between 2011 and 2014. Mayo Clin Proc 2015;90:1600-13.

9. Levac D, Colquhoun $\mathrm{H}, \mathrm{O}$ 'Brien KK. Scoping studies: advancing the methodology. Implement Sci 2010;5:69.

10. Arksey H, O'Malley L. Scoping studies: towards a methodological framework. Int J Soc Res Methodol 2005;8:19-32.

11. McGowan J, Sampson M, Salzwedel DM, et al. PRESS peer review of electronic search strategies: 2015 guideline statement. J Clin Epidemiol 2016;75:40-6.

12. The Joanna Briggs Institute. The Joanna Briggs Institute Reviewers' manual 2015: methodology for JBI scoping reviews. Joanne Briggs Inst 2015:1-24. 